\title{
Infinitesimal Takesaki duality of Hamiltonian vector fields on a symplectic manifold
}

\author{
Katsunori Kawamura 由 \\ Research Institute for Mathematical Sciences \\ Kyoto University, Kyoto 606,Japan
}

November 20, 2018.

\begin{abstract}
For an infinitesimal symplectic action of a Lie algebra $\mathfrak{g}$ on a symplectic manifold, we construct an infinitesimal crossed product of Hamiltonian vector fields and Lie algebra $\mathfrak{g}$. We obtain its second crossed product in case $\mathfrak{g}=\mathbf{R}$ and show an infinitesimal version for a theorem type of Takesaki duality.
\end{abstract}

\section{Introduction}

By an application of a functional representation for $\mathcal{L}(\mathcal{H})$ [5], any von Neumann algebra is faithfully represented as functions on a projective Hilbert space $\mathcal{P}(\mathcal{H})$ by $*$-product as an unital $\mathrm{W}^{*}$-algebra. Its non commutativity comes from the symplectic structure of $\mathcal{P}(\mathcal{H})$ induced by the fundamental form of a Kähler metric of $\mathcal{P}(\mathcal{H})$. These results are showed in section 2 . In this view, we expect that several structure of von Neumann algebra come from symplectic geometry.

Our aim is a study how the structures of von Neumann algebra is recognized as the symplectic geometry on $\mathcal{P}(\mathcal{H})$.

\footnotetext{
1 e-mail : kawamura@kurims.kyoto-u.ac.jp.
} 
For example, by [1] the $*$-product of functions of some Kähler manifold $M$ is depend on its holomorphic sectional curvature $c$ of $M$ by the deformation parameter $1 / c$. And the functional representation for von Neumann algebra is the case of $c=1$. And the represented commutator of von Neumann algebra becomes just Poisson bracket of functions multiplying $\sqrt{-1}$. In other words, von Neumann algebra is a special kind of " symplectic algebra ". ( See corollary 2.1, too. )

In this paper, we define an infinitesimal crossed product by the semi direct product of Lie algebras and show Takesaki duality type theorem for some dynamical system on a symplectic manifold.

Theorem 1.1 (Infinitesimal Takesaki duality)

Let $M$ be a symplectic manifold and $(M, \mathbf{R}, \beta)$ an infinitesimal symplectic dynamical system induced by some symplectic dynamical system, $\mathfrak{X}_{H}(M)$ the Lie algebra of all Hamiltonian vector fields on $M$. Then an isomorphism of Lie algebras follows

$$
\left(\mathfrak{X}_{H}(M) \rtimes_{\beta} \mathbf{R}\right) \rtimes_{\hat{\beta}} \mathbf{R} \cong \mathfrak{X}_{H}(M) \rtimes_{\delta} H_{1}
$$

where $\hat{\beta}$ is the dual action of $\beta$ and $H_{1}$ is the Heisenberg Lie algebra with two generators $a, a^{\dagger}$ over $\mathbf{C}$ which satisfying $\left[a, a^{\dagger}\right]=I$. The left hand side is semi direct product of Lie algebras induced by a derivative action $\delta: H_{1} \rightarrow \operatorname{End}\left(\mathfrak{X}_{H}(M)\right)$ such that $\delta_{a} X_{f}=\frac{1}{\sqrt{2}} X_{\beta_{1} f}, \delta_{a^{\dagger}} X_{f}=-\frac{1}{\sqrt{2}} X_{\beta_{1} f}$ for $X_{f} \in \mathfrak{X}_{H}(M), f \in C^{\infty}(M)$.

By the functional representation for von Neumann algebra, this theorem is recognized as a generalization of Takesaki duality for non associative algebra related to symplectic structure of symplectic manifold. About Takesaki duality, see [11].

\section{A functional representation for non com- mutative von Neumann algebras}

We construct a functional representation of von Neumann algebras as follows.

Let $\mathcal{H}$ be a Hilbert space and $\mathcal{L}(\mathcal{H})$ the set of all bounded linear operators on $\mathcal{H}$. The following result are obtained by Cirelli, Lanzavecchia, ManiàPizzocchero et al. 
Theorem 2.1 (A functional representation for $\mathcal{L}(\mathcal{H})$ 可)

(i). $\mathcal{P}(\mathcal{H}) \equiv(\mathcal{H}-\{0\}) / \mathbf{C}^{\times}$becomes a Kähler manifold $(\mathcal{P}(\mathcal{H}), g)$ by the inhomogeneous coordinate and the Fubini-study type metric $g$.

(ii). A set of functions $\mathcal{K}(\mathcal{P}(\mathcal{H})) \equiv\left\{f \in C^{\infty}(\mathcal{P}(\mathcal{H})): D^{2} f=0, \bar{D}^{2} f=0\right\}$ becomes a $C^{*}$-algebra by a $*$-product

$$
(f * l)(\mathbf{x}) \equiv f(\mathbf{x}) l(\mathbf{x})+\partial_{\mathbf{x}} f\left(\operatorname{grad}_{\mathbf{x}} l\right) \quad f, l \in \mathcal{K}(\mathcal{P}(\mathcal{H})), \mathbf{x} \in \mathcal{P}(\mathcal{H})
$$

, a*-involution

$$
f^{*} \equiv \text { complex conjugate } \bar{f} \text { of } f
$$

and a $C^{*}$-norm

$$
\|f\| \equiv \sup _{\mathbf{x} \in \mathcal{P}(\mathcal{H})}|(\bar{f} * f)(\mathbf{x})|^{1 / 2} .
$$

Here $D, \bar{D}$ are the holomorphic and anti-holomorphic part of covariant derivative, and $\operatorname{grad} f$ is the holomorphic part of complexified gradient of $f$ by the Kähler metric $g$.

(iii). $\mathcal{L}(\mathcal{H}) \cong \mathcal{K}(\mathcal{P}(\mathcal{H}))$ as a $C^{*}$-algebra.

Proof. (i) See [3]. ( Topology is induced by inhomogeneous coordinate. Differential structure is defined by Frechet differential.) (ii) and (iii) is in the section 2 of [可]. For $A \in \mathcal{L}(\mathcal{H})$, let $f_{A}([x]) \equiv<x \mid A x>$ for $[x] \in \mathcal{P}(\mathcal{H})$, $x \in \mathcal{H},\|x\|=1$.

Remark 2.1 In this theorem, Kähler manifold means a Hilbert manifold with a nondegenerate hermitian metric which fundamental form is closed. And if $\operatorname{dim} \mathcal{H}=n \in \mathbf{N}$, then $\mathcal{P}(\mathcal{H})$ becomes ordinary projective space $\mathbf{C} P^{n-1}$ with the standard Kähler structure.

By the definition of Poison bracket $\{\cdot, \cdot\}$ of the fundamental form of $\mathcal{P}(\mathcal{H})$, the following relation holds

$$
f * l-l * f=\sqrt{-1}\{f, l\}
$$

for $f, l \in \mathcal{K}(\mathcal{P}(\mathcal{H}))$. 
Definition 2.1 (i). For a subset $\mathcal{S} \subset \mathcal{K}(\mathcal{P}(\mathcal{H}))$, the Poisson commutant $\mathcal{S}^{c} \equiv\{f \in \mathcal{K}(\mathcal{P}(\mathcal{H})):\{f, l\}=0$ for $l \in \mathcal{S}\}$.

(ii). The strong Kähler topology of $\mathcal{K}(\mathcal{P}(\mathcal{H}))$ is defined by pointwise convergence $f_{\lambda} \rightarrow f$ of net $\left\{f_{\lambda}\right\}$ of functions in $\mathcal{K}(\mathcal{P}(\mathcal{H}))$ by $\left(\bar{f}_{\lambda} * f_{\lambda}\right)(p) \rightarrow$ $(\bar{f} * f)(p)$ for each $p \in \mathcal{P}(\mathcal{H})$.

(iii). The weak Kähler topology of $\mathcal{K}(\mathcal{P}(\mathcal{H}))$ is defined by pointwise convergence $f_{\lambda} \rightarrow f$ of net $\left\{f_{\lambda}\right\}$ of functions by $f_{\lambda}(p) \rightarrow f(p)$ for each $p \in \mathcal{P}(\mathcal{H})$.

Remark if we say " subalgebra " of $\mathcal{K}(\mathcal{P}(\mathcal{H}))$, it means a subalgebra by *-product in theorem 2.1 (ii).

By using theorem 2.1, we construct a functional faithful representation for a non commutative von Neumann algebras.

Corollary 2.1 (A functional representation for a von Neumann algebra) For each von Neumann algebra acting $\mathcal{R}$ on a Hilbert space $\mathcal{H}$, there is a subalgebra $\mathcal{S}$ of $\mathcal{K}\left(\mathcal{P}(\mathcal{H})\right.$ ) such that $\mathcal{S}^{c c}=\mathcal{S}$ and closed under complex conjugate and it is isomorphic to $\mathcal{R}$ as an unital $W^{*}$-algebra by the strong Kähler topology in $\mathcal{K}(\mathcal{P}(\mathcal{H}))$.

Proof. By theorem 2.1 (iii), for a von Neumann algebra $\mathcal{R}$, let $\mathcal{S} \subset \mathcal{K}(\mathcal{P}(\mathcal{H})$ ) be a range of the functional representation of $\mathcal{R} \subset \mathcal{L}(\mathcal{H})$. Then $\mathcal{S}$ becomes *-subalgebra by theorem 2.1 (iii) and by the relation of commutator and Poisson bracket in the last argument, it satisfies $\mathcal{S}^{c c}=\mathcal{S}$.

Because of definition of norm in $\mathcal{K}(\mathcal{P}(\mathcal{H})),\|A x\|=\left|\left(\bar{f}_{A} * f_{A}\right)(\mathbf{x})\right|$ for $A \in \mathcal{R}, x \in \mathcal{H}, \mathbf{x}=[x] \in \mathcal{P}(\mathcal{H})$ and $f_{A}$ is a function in $\mathcal{S}$ corresponding to $A$. The topology of $\mathcal{S}$ related to strong operator topology of $\mathcal{R}$ is just equal to the strong Kähler topology.

Corollary 2.2 For a *-subalgebra $\mathcal{S}$ with $1_{M}$ of $\mathcal{K}(\mathcal{P}(\mathcal{H})), \mathcal{S}$ is weak Kähler closed if and only if $\mathcal{S}=\mathcal{S}^{c c}$.

Proof. By corollary 2.1, $\mathcal{S}=\mathcal{S}^{c c}$ if and only if there is a related von Neumann algebra $\mathcal{R}$ and it is weak operator closed. By definition of weak Kähler topology, it is equivalent to weak operator topology $\mathcal{R}$. Since a unital $*-$ algebra $\mathcal{R}$ of $\mathcal{L}(\mathcal{H})$ is weak operator closed if and only if it is strong operator closed, $\mathcal{S}$ is weak Kähler closed if and only if $\mathcal{S}$ is strong Kähler closed. 
Comparison with non-commutative geometry In the theory of noncommutative geometry by A.Connes [6], operator algebras are treated as like some function spaces on "virtual " manifolds. there are many applications and similar the theory of results for the theory of original geometry [7].

But we cannot view non-commutative manifold which related to noncommutative operator algebras.

Corollary 2.1 gives some answers for this problems as follows: By the functional representation, any von Neumann algebra acting on a Hilbert space $\mathcal{H}$ is identified with function $*$-sub algebra $\mathcal{S}$ of $\mathcal{K}(\mathcal{P}(\mathcal{H}))$ such that it is closed under bi-commutator in $\mathcal{K}\left(\mathcal{P}(\mathcal{H})\right.$ ). ( About the case of $\mathrm{C}^{*}$-algebras, see [5]. ) By an equation 2.1, the commutator of functions is given by Poisson bracket on $\mathcal{P}(\mathcal{H})$. Since Poisson bracket is defined by symplectic form ( fundamental form ) of $\mathcal{P}(\mathcal{H})$.

We can conclude that " non commutativity of von Neumann algebras comes from skew symmetry of symplectic form on $\mathcal{P}(\mathcal{H})$ ".

symplectic structure is well known and fundamental non commutative structure of non " virtual " geometry. This is a solution for how noncommutative structure of operator algebra comes from real geometry.

Remark 2.2 (i). Usually, von Neumann algebra is considered as "non commutative $L_{p}$-space " or some kind of dual of measure space. So someone may feel that measurable functions on measure spaces are more suitable for a functional representation of von Neumann algebra rather than smooth functions on a smooth manifold. But we don't touch such a way of view in this paper.

(ii). For a unital $\mathrm{C}^{*}$-algebra $\mathfrak{A}$ acting on a Hilbert space $\mathcal{H}$, its enveloping von Neumann algebra $\overline{\mathfrak{A}}$ is the strong operator closure of it. So someone may feel that a functional representation is given by extension of Cirelli-Manià-Pizzocchero functional representation by taking closure of it [5]. But it does not stands because generally Cirelli-ManiàPizzocchero functional representation is not strong operator continuous.

(iii). The relations of so called $*$-deformation of functions over a symplectic manifold and $\mathrm{C}^{*}$-algebra are studied by Riefell [10]. In our paper, we use a $*$-product as similar as $*$-deformation, but there are several differences : (a) There is no deformation parameter in appearance in our 
theory. In [4], deformation parameter is equal to the inverse of holomorphic sectional curvature of Kähler manifold. In this case deformation parameter is fixed to 1 because projective space has holomorphic sectional curvature 1. (b) We treat only first-order deformation. Usually, the deformation algebra is the algebra of formal power series with coefficient of functions on s symplectic manifold. But our case, there is no term except 0 -th and 1-st orders. (c) Our deformed functional space is not closed usual point wise product of functions. (d) We don't deform operator algebras but represent as function space.

\section{Infinitesimal symplectic dynamical system}

We define the infinitesimal symplectic dynamical system and introduce some example of it. -text follows this line- Let $M$ be a symplectic manifold i.e. a smooth manifold with nondegenerate closed 2-form $\omega$ and the set $\mathfrak{X}(M)$ of all smooth vector fields on $M . \operatorname{Symp}(M)$ is the group of all symplectic diffeomorphisms on $M$. For $f \in C^{\infty}(M)$, a vector field $X_{f}$ is called Hamiltonian if it satisfies $\omega_{p}\left(\left(X_{f}\right)_{p}, u\right)=d_{p} f(u)$ for any $p \in M$ and any tangent vector $u$ at $p$. It is well defined by non degeneracy of $\omega$. We denote the set of all Hamiltonian vector fields on $M$ by $\mathfrak{X}_{H}(M)$. Then $\mathfrak{X}_{H}(M)$ is a Lie subalgebra of $\mathfrak{X}(M)$ by the commutator $[\cdot, \cdot]$ of vector fields. and $\left(C^{\infty}(M),\{\cdot, \cdot\}\right) \rightarrow\left(\mathfrak{X}_{H}(M),[\cdot, \cdot]\right), f \mapsto-X_{f}$ is surjective Lie homomorphism with kernel $\mathrm{C}_{M}$ where $\{\cdot, \cdot\}: C^{\infty}(M) \times C^{\infty}(M) \rightarrow C^{\infty}(M)$ is the Poisson bracket defined by $\{f, l\}_{p} \equiv \omega_{p}\left(\left(X_{f}\right)_{p},\left(X_{l}\right)_{p}\right)$ for $f, l \in C^{\infty}(M)$ at $p \in M$ [ $\left.\mathbb{\theta}\right]$.

Let $G$ be a Lie group with its Lie algebra $\mathfrak{g}$.

Definition 3.1 (i). (M, G, $\alpha)$ is a symplectic dynamical system if $\alpha: G \rightarrow$ $\operatorname{Symp}(M)$ is a symplectic action of $G$ on $M$ which is smooth $G \ni g \mapsto$ $\alpha_{g}(p) \in M$ at each point $p \in M$.

(ii). $(M, \mathfrak{g}, \beta)$ is an infinitesimal symplectic dynamical system if $\beta: \mathfrak{g} \rightarrow$ $\mathfrak{X}(M)$ is Lie homomorphism such that $\beta_{X}\{f, l\}=\left\{\beta_{X} f, l\right\}+\left\{f, \beta_{X} l\right\}$ for any $f, l \in C^{\infty}(M)$ and $X \in \mathfrak{g}$.

Remark 3.1 For a given symplectic dynamical system $(M, G, \alpha),\left(\beta_{X} f\right)(p) \equiv$ 
$\left.\frac{d}{d t}\left(\alpha_{\exp (t X)}^{*} f\right)(p)\right|_{t=0}$ for $p \in M$ becomes an infinitesimal symplectic dynamical system. The reason why we treat infinitesimal symplectic dynamical systems is follows : Ordinary crossed product in the theory of operator algebras is an associative algebra generated by an associative algebra and a group algebra. But we treat $C^{\infty}(M)$ as a Lie algebra by its Poisson bracket. So we consider to construct a crossed product of Lie algebras as a Lie algebra by using semi direct product of Lie algebras. Relation between von Neumann algebra and infinitesimal symplectic dynamical system is explained later.

Example 3.1 (i) $G=\mathbf{R}_{+} \equiv\left\{e^{t}: t \in \mathbf{R}\right\}, \mathfrak{g}=\mathbf{R}$. Then $\alpha$ is symplectic flow on $M$.

(ii) If $M$ is a Kähler manifold and $\alpha: G \rightarrow \operatorname{Iso}_{h}(M)$ is holomorphic Kähler isometric action, then automatically $\alpha$ becomes symplectic action for the fundamental form of the Kähler metric. So $(M, G, \alpha)$ becomes symplectic dynamical system. So, if $G$ is a Lie subgroup of Kähler isometries which are holomorphic gives a symplectic dynamical system.

(iii) Specially simply connected, connected complete Kähler manifold $M$ with non zero constant holomorphic sectional curvature $c$ is considered in some geometric generalization of quantum mechanics ([1], [2], [4]). Its symplectic dynamical system $\left(M, \mathbf{R}_{+}, \alpha\right)$ induces the generalized Schrödinger equation on $M$. In this case, we need to remove a condition of smoothness of action $\mathbf{R}_{+} \ni x \mapsto \alpha_{x}(p)$ for $p \in M$ without some region when it is necessary to treat unboundedness of Hamiltonian.

(iv) If $M=\mathcal{P}(\mathcal{H})$ over ( possibly infinite dimensional ) Hilbert space $\mathcal{H}$, its symplectic dynamical system is related to $\mathrm{W}^{*}$-dynamical system $(\mathcal{R}, G, \sigma)$ ( appendix A ) by a functional representation of a von Neumann algebra $\mathcal{R}$ on $\mathcal{P}(\mathcal{H})$ ( appendix ?? ) where $\sigma$ is an action of locally compact group $G$ on $\mathcal{R}$ as *-automorphisms implemented by unitary representation of $G$ on $\mathcal{H}$.

In the case $G$ is a Lie group with the Lie algebra $\mathfrak{g}$ and there is a dense linear subspace $\mathcal{D} \subset \mathcal{H}$ such that $d U: \mathfrak{g} \rightarrow \operatorname{End}(\mathcal{D})$ is the infinitesimal representation of $\mathfrak{g}$ induced by the smooth representation $U$ of $G$ on $\mathcal{D}$.

Let $d \sigma_{X} \equiv \operatorname{ad}_{d U_{X}}$ for $x \in \mathfrak{g}$. Then $d \sigma: \mathfrak{g} \rightarrow \operatorname{End}(\mathcal{R})$ becomes representation of $\mathfrak{g}$ where $\mathcal{R}$ is restricted on $\mathcal{D}$. By the functional representation $\mathcal{S}=\left\{f_{A}: A \in \mathcal{R}\right\}$ of $\mathcal{R}$, define $\beta_{X}\left(f_{A}\right) \equiv f_{d \sigma_{X}(A)}$ for $X \in \mathfrak{g}$. Then $(\mathcal{P}(\mathcal{H}), \mathfrak{g}, \beta)$ becomes an infinitesimal dynamical system with function space $\mathcal{S} \subset \mathcal{K}(\mathcal{P}(\mathcal{H}))$. 


\section{Infinitesimal symplectic covariant system}

We define infinitesimal symplectic covariant system and introduce some example, and define crossed product of Hamiltonian vector fields.

Let $(M, \mathfrak{g}, \beta)$ be an infinitesimal symplectic dynamical system.

Definition $4.1(\pi, \Lambda)$ is an infinitesimal covariant system of $(M, \mathfrak{g}, \beta)$ if there is a vector space $V, \pi:\left(C^{\infty}(M),\{\cdot, \cdot\}\right) \rightarrow \operatorname{End}(V), \Lambda: \mathfrak{g} \rightarrow \operatorname{End}(V)$ are Lie homomorphisms and they satisfy $\left[\Lambda_{X}, \pi(f)\right]=\pi\left(\beta_{X} f\right)$ for each $X \in$ $\mathfrak{g}, f \in C^{\infty}(M)$.

Clearly, infinitesimal symplectic covariant systems come from symplectic covariant systems by differential $\mathbf{R} \ni t \mapsto \exp (t X) \in G$.

Once more we remark that the reason why we use infinitesimal form is in order to construct crossed product of Lie algebras of $\left(C^{\infty}(M),\{\cdot, \cdot\}\right)$ and some Lie algebra because von Neumann algebra is a sub Lie algebra of $C^{\infty}(\mathcal{P}(\mathcal{H}))$ by Poisson bracket.

Example 4.1 (i). Let

$$
V \equiv C^{\infty}(M) \quad \pi(f) l \equiv(\operatorname{ad} f)(l)=-X_{f} l=\{f, l\} \quad \Lambda_{X} l \equiv \beta_{X} l
$$

for $X \in \mathfrak{g}, f, l \in C^{\infty}(M)$. Then $(\pi, \Lambda)$ becomes an infinitesimal symplectic covariant system by Leibniz rule of $\beta_{X}$.

(ii). Let $(M, G, \alpha)$ be a symplectic dynamical system which induces a given infinitesimal symplectic dynamical system $(M, \mathfrak{g}, \beta)$. Let

$$
\begin{gathered}
V \equiv C^{\infty}(M) \otimes C^{\infty}(G) \\
(\pi(f)(l \otimes \chi))(p, g) \equiv\left\{\alpha_{g^{-1}}^{*} f, l\right\}_{p} \chi(g) \quad \Lambda_{X} \equiv I \otimes l_{X}
\end{gathered}
$$

for $X \in \mathfrak{g}, f, l \in C^{\infty}(M), \chi \in C^{\infty}(G)$ where $\otimes$ is algebraic tensor over $\mathbf{C}$ and $\left.\left(l_{X} \chi\right)(g) \equiv \frac{d}{d t} \chi(\exp (-t X) \cdot g)\right|_{t=0}$. Then $(\pi, \Lambda)$ becomes an infinitesimal symplectic covariant system. As the same way, $\tilde{V} \equiv$ $C^{\infty}(M \times G)$ gives an infinitesimal symplectic covariant system. If we take the right regular representation,

$$
\left(\pi^{\prime}(f)(l \otimes \chi)\right)(p, g) \equiv\left\{\alpha_{g}^{*} f, l\right\}_{p} \chi(g) \quad \Lambda_{X}^{\prime} \equiv I \otimes r_{X}
$$

where $X \in \mathfrak{g}, f, l \in C^{\infty}(M), \chi \in C^{\infty}(G),\left.\left(r_{X} \chi\right)(g) \equiv \frac{d}{d t} \chi(g \cdot \exp (t X))\right|_{t=0}$. Then $\left(\pi^{\prime}, \Lambda^{\prime}\right)$ it becomes an infinitesimal symplectic covariant system, too. 
Remark 4.1 In the theory of operator algebras, a covariant system is a couple of representations of algebra and group defined on a same Hilbert space. But in the infinitesimal case, we can not use Hilbert space generally. For example, consider time development of unbounded Hamiltonian $H$, then its differential is not defined on whole Hilbert space. As same as in TomitaTakesaki theory, $H=\log \Delta$ is unbounded operator generally where $\Delta$ is a modular operator. So generator of action of $\mathbf{R}$ is not able to defined on whole of $L_{2}(\mathbf{R})$.

In order to define a crossed product of an infinitesimal symplectic dynamical system $(M, \mathfrak{g}, \beta)$ by an infinitesimal covariant system $(\pi, \Lambda)$, we review the semi direct product of Lie algebras.

For two Lie algebras $\mathfrak{g}_{1}, \mathfrak{g}_{2}$, consider a representation $\delta: \mathfrak{g}_{2} \rightarrow \operatorname{End}\left(\mathfrak{g}_{1}\right)$ such that $\delta_{b}$ is a derivation that is $\delta_{b}([v, w])=\left[\delta_{b} v, w\right]+\left[v, \delta_{b} w\right]$ for $b \in$ $\mathfrak{g}_{2}, v, w \in \mathfrak{g}_{1}$. We call such $\delta$ by derivative action of $\mathfrak{g}_{2}$ on $\mathfrak{g}_{1}$.

The semi direct product $\mathfrak{g}_{1} \rtimes_{\delta} \mathfrak{g}_{2}$ is a Lie algebra which is equal to direct sum $\mathfrak{g}_{1} \oplus \mathfrak{g}_{2}$ as a linear space and has a new Lie bracket $[b, v] \equiv \delta_{b} v$ for $b \in \mathfrak{g}_{2}, v \in \mathfrak{g}_{1}$ and other Lie brackets are same as that of $\mathfrak{g}_{1}, \mathfrak{g}_{2}$ respectively.

For an infinitesimal symplectic dynamical system $(M, \mathfrak{g}, \beta), \beta: \mathfrak{g} \rightarrow$ $\mathfrak{X}(M) \subset \operatorname{End}\left(C^{\infty}(M)\right)$ is a derivative action. For an infinitesimal covariant system $(\pi, \Lambda)$ of $(M, \mathfrak{g}, \beta)$, if let $\gamma_{\xi} \equiv \operatorname{ad} \xi$ for $\xi \in \Lambda(\mathfrak{g})$, then $\gamma: \Lambda(\mathfrak{g}) \rightarrow$ $\operatorname{End}\left(\pi\left(C^{\infty}(M)\right)\right.$ is a derivative action, too. So, we can construct two semi direct products $C^{\infty}(M) \rtimes_{\beta} \mathfrak{g}$ and $\pi\left(C^{\infty}(M)\right) \rtimes_{\gamma} \Lambda(\mathfrak{g})$.

If $(\pi, \Lambda)$ is the infinitesimal covariant system in previous example (ii), then $\pi\left(C^{\infty}(M)\right) \cong \mathfrak{X}_{H}(M)$ because kernel of $\pi=\mathbf{C} 1_{M}$ and $\Lambda(\mathfrak{g}) \cong \mathfrak{g}$. Therefore

Definition 4.2 (Crossed product of Hamiltonian vector field) $\mathfrak{X}_{H}(M) \rtimes_{\beta} \mathfrak{g} \equiv$ $\pi\left(C^{\infty}(M)\right) \rtimes_{\gamma} \Lambda(\mathfrak{g})$ for the infinitesimal covariant system in example 4.1 (ii).

\section{Second crossed product}

To state the duality theorem, we need the definition of second crossed product and restrict $G$ to an abelian Lie group which dual becomes Lie algebras,too because the duality theorem comes from comes from duality of abelian group and we treat a smooth class of group. Then we treat only $G=\mathbf{R}_{+}$. Let $(M, \mathbf{R}, \beta)$ be an infinitesimal dynamical system. For $\mathcal{L}_{1} \equiv \mathfrak{X}_{H}(M) \rtimes_{\beta} \mathbf{R}$, we 
define a dual infinitesimal covariant system. Remark now $G=\mathbf{R}_{+}, \mathfrak{g}=\mathbf{R}$. But we identify $G$ and $\mathfrak{g}$ in this case from now.

Let $V_{1} \equiv C^{\infty}(M) \otimes C^{\infty}(\mathbf{R})$ and define an operator $W_{s}: V_{1} \rightarrow V_{1}$ by

$$
\left(W_{s} \Psi\right)(p, t) \equiv \exp (\sqrt{-1} t s) \Psi(p, t)
$$

for $\Psi \in V_{1}, s, t \in \mathbf{R}$ and

$$
\left.\hat{\alpha}_{s} \equiv \operatorname{Ad} W_{s} \quad \hat{\beta}_{s} \equiv \frac{d}{d x} \hat{\alpha}_{s x}\right|_{x=0}
$$

on $\mathcal{L}_{1}$.

Let $\left(w_{s} \chi\right)(t) \equiv \sqrt{-1} s t \chi(t)$ for $\chi \in C^{\infty}(\mathbf{R})$ and $t, s \in \mathbf{R}$. Then $((I \otimes$ $\left.\left.w_{s}\right) \Psi\right)(p, t)=\left.\frac{d}{d x}\left(W_{s} \Psi\right)(p, t)\right|_{x=0}$, and $\hat{\beta}_{s}(R)=\left[I \otimes w_{s}, R\right]$ for $s \in \mathbf{R}, R \in \mathcal{L}_{1}$. Furthermore by Jacobi identity of bracket, $\hat{\beta}: \mathbf{R} \rightarrow \operatorname{End}\left(\mathcal{L}_{1}\right)$ becomes a derivative action.

Lemma 5.1 (i). $\hat{\beta}_{s}(\pi(f))=0$ for $f \in C^{\infty}(M), s \in \mathbf{R}$.

(ii). $\hat{\beta}_{s}\left(\Lambda_{t}\right)=\sqrt{-1} s t I$

Proof (i) By definition, $\hat{\alpha}_{s}(\pi(f))=\pi(f)$ for any $f \in C^{\infty}(M), s \in \mathbf{R}$. So, the statement follows.

(ii) $\left(\hat{\alpha}_{s}\left(\Lambda_{t}\right)(\Psi)\right)(p, r)$

$$
\begin{aligned}
& =\left(W_{s} \Lambda_{t} W_{-s} \Psi\right)(p, r) \\
& =e^{\sqrt{-1} r s}\left(\Lambda_{t} W_{-s} \Psi\right)(p, r) \\
& =\left.e^{\sqrt{-1} r s} \frac{d}{d x}\left(W_{-s} \Psi\right)(p, r-t x)\right|_{x=0} \\
& =\left.e^{\sqrt{-1} r s} \frac{d}{d x} e^{-\sqrt{-1}(r-t x) s} \Psi(p, r-t x)\right|_{x=0} \\
& =\sqrt{-1} t s \Psi(p, r)+\left.\frac{d}{d x} \Psi(p, r-t x)\right|_{x=0} .
\end{aligned}
$$

So $\hat{\alpha}_{s}\left(\Lambda_{t}\right)=\Lambda_{t}+\sqrt{-1} s t I$. Therefore, the statement follows.

By this lemma, $\hat{\alpha}_{t}\left(\left[R, R^{\prime}\right]\right)=\left[R, R^{\prime}\right]$ for each $R, R^{\prime} \in \mathcal{L}_{1}, t \in \mathbf{R}$.

Let $V_{2} \equiv V_{1} \otimes C^{\infty}(\mathbf{R})$. Define $\hat{\pi}: \mathcal{L}_{1} \rightarrow \operatorname{End}(V)$ and $\hat{\Lambda}: \mathbf{R} \rightarrow \operatorname{End}(V)$ by $\left.(\hat{\pi}(R) \Phi)(p, s, r) \equiv\left(\hat{\alpha}_{-r}(R) \otimes I\right) \Phi\right)(p, s, r)$ and $\hat{\Lambda}_{t}=I \otimes I \otimes l_{t}$ for $r, s, t \in$ $\mathbf{R}, R \in \mathcal{L}_{1}, \Phi \in V_{2}$.

Lemma 5.2 (i). $\left[\hat{\Lambda}_{t}, \hat{\pi}(R)\right]=\hat{\pi}\left(\hat{\beta}_{t}(R)\right)$ on $V_{2}$ for $t \in \mathbf{R}, R \in \mathcal{L}_{1}$

(ii). $\hat{\pi}(\pi(f))=\pi(f) \otimes I$ for $f \in C^{\infty}(M)$ 
(iii). $\hat{\pi}\left(\Lambda_{t}\right)=\Lambda_{t} \otimes I-I \otimes I \otimes w_{t}$ for $t \in \mathbf{R}$ for $t \in \mathbf{R}$.

$\operatorname{Proof}(\mathrm{i})$ For $\Phi \in V_{2}$,

$$
\begin{aligned}
&\left(\left[\hat{\Lambda}_{t}, \hat{\pi}(R)\right] \Phi\right)(p, r, s) \\
&=\left.\frac{d}{d x}(\hat{\pi}(R) \Phi)(p, r, s-x t)\right|_{x=0} \\
&=-\left\{\left(\hat{\alpha}_{-s}(R) \otimes I\right) \hat{\Lambda}_{t} \Phi\right\}(p, r, s) \\
&=\left.\frac{d}{d x}\left\{\left(\hat{\alpha}_{-(s-x t)}(R) \otimes I\right) \Phi\right\}(p, r, s-x t)\right|_{x=0} \\
&-\left.\left\{\left(\hat{\alpha}_{-s}(R) \otimes I\right) \frac{d}{d x} \Phi\right\}(p, r, s-x t)\right|_{x=0} \\
&=\left\{\left(\left.\hat{\alpha}_{-s}\left(\frac{d}{d x} \hat{\alpha}_{x t}(R)\right)\right|_{x=0} \otimes I\right) \Phi\right\}(p, r, s) \\
&=\left.\left\{\hat{\alpha}_{-s}\left(\hat{\beta}_{t}(R)\right) \otimes I\right) \Phi\right\}(p, r, s) \\
&=\left\{\hat{\pi}\left(\hat{\beta}_{t}(R)\right) \Phi\right\}(p, r, s)
\end{aligned}
$$

(ii) By proof of lemma 5.1 (i), it follows.

(iii) $\left(\hat{\pi}\left(\Lambda_{t}\right) \Phi\right)(p, r, s)=\left(\left(\hat{\alpha}_{-s}\left(\Lambda_{t}\right) \otimes I\right) \Phi\right)(p, r, s)$

By proof of lemma 5.1 (ii),

$=\left\{\left(\left(\Lambda_{t}-\sqrt{-1} s t\right) \otimes I\right) \Phi\right\}(p, r, s)$

$=\left(\left(\Lambda_{t} \otimes I-I \otimes I \otimes w_{t}\right) \Phi\right)(p, r, s)$

We define second crossed product $\left(\mathfrak{X}_{H}(M) \rtimes_{\beta} \mathbf{R}\right) \rtimes_{\hat{\beta}} \mathbf{R}$ by semi direct product $\hat{\pi}\left(\mathfrak{X}_{H}(M) \rtimes_{\beta} \mathbf{R}\right) \rtimes_{\text {ad } \hat{\Lambda}} \mathbf{R}$ of Lie algebras $\mathfrak{X}_{H}(M) \rtimes_{\beta} \mathbf{R}$ and $\mathbf{R}$.

\section{Infinitesimal Takesaki duality}

For an infinitesimal symplectic dynamical system $(M, \mathbf{R}, \beta)$, we show the structure of second crossed product $\left(\mathfrak{X}_{H}(M) \rtimes_{\beta} \mathbf{R}\right) \rtimes_{\hat{\beta}} \mathbf{R}$. In order to use the duality of abelian group $\mathbf{R}=\hat{\mathbf{R}}$, we restrict the representation space to $C^{\infty}(M) \otimes \mathcal{S}(\mathbf{R}) \otimes \mathcal{S}(\mathbf{R})$ where $\mathcal{S}(\mathbf{R}) \subset C^{\infty}(\mathbf{R})$ is the set of Schwartz class functions over $\mathbf{R}$ that is, they satisfy $\lim _{|t| \rightarrow \infty}|t|^{n-1}\left|\partial^{m-1} f(t)\right|=0$ for any $n, m \in \mathbf{N}$.

Recall the Heisenberg Lie algebra $H_{1}$ is a Lie algebra with a couple of generators $a, a^{\dagger}$ which satisfying $\left[a, a^{\dagger}\right]=I$ over $\mathbf{C}$.

By these preparations, we can state our main theorem.

Theorem 6.1 (Infinitesimal Takesaki duality) For an infinitesimal symplectic dynamical system $(M, \mathbf{R}, \beta)$ induced by a symplectic dynamical system $(M, \mathbf{R}, \alpha)$ 
(i). there is a derivative action $\delta: H_{1} \rightarrow \operatorname{End}\left(\mathfrak{X}_{H}(M)\right)$ such that

$$
\delta_{a} X_{f}=\frac{1}{\sqrt{2}} X_{\beta_{1} f}, \quad \delta_{a^{\dagger}} X_{f}=-\frac{1}{\sqrt{2}} X_{\beta_{1} f} \quad \text { for } f \in C^{\infty}(M) .
$$

(ii). For the derivative action in (i), there is an isomorphism of Lie algebras

$$
\left(\mathfrak{X}_{H}(M) \rtimes_{\beta} \mathbf{R}\right) \rtimes_{\hat{\beta}} \mathbf{R} \cong \mathfrak{X}_{H}(M) \rtimes_{\delta} H_{1}
$$

where the second crossed product at left hand side is represented on $C^{\infty}(M) \otimes \mathcal{S}(\mathbf{R}) \otimes \mathcal{S}(\mathbf{R})$.

Proof (i) The existence of $\delta$ is showed in the proof of (ii).

(ii) We prove this statement by tracing the proof of Takesaki duality theorem of von Neumann algebra ( vol. II, theorem 13.2.9 in [9]).

Let

$$
\begin{gathered}
\mathcal{L}_{1} \equiv \mathfrak{X}_{H}(M) \rtimes_{\beta} \mathbf{R}, \quad \mathcal{L}_{2} \equiv\left(\mathfrak{X}_{H}(M) \rtimes_{\beta} \mathbf{R}\right) \rtimes_{\hat{\beta}} \mathbf{R} \\
V_{0} \equiv C^{\infty}(M) \otimes \mathcal{S}(\mathbf{R}), \quad V_{3} \equiv C^{\infty}(M) \otimes \mathcal{S}(\mathbf{R}) \otimes \mathcal{S}(\mathbf{R})
\end{gathered}
$$

By definition, $\mathcal{L}_{1}$ is generated by

$$
\pi(f) \quad \Lambda_{s} \quad f \in C^{\infty}(M), s \in \mathbf{R}
$$

Let $U_{1}: V_{0} \rightarrow V_{0}$ be $\left(U_{1} \Psi\right)\left(p, s^{\prime}\right) \equiv \Psi\left(\alpha_{-s^{\prime}}(p), s^{\prime}\right)$ for $\Psi \in V_{0}$. Then $U_{1}$ becomes a linear automorphism on $V_{0}$ and $U_{1} \pi(f) U_{1}^{-1}=-X_{f} \otimes I, U_{1} \Lambda_{s} U_{1}^{-1}=$ $\beta_{s} \otimes I+I \otimes l_{s}$. where $\left.\left(l_{s} \chi\right)\left(p, s^{\prime}\right) \equiv \frac{d}{d x} \chi\left(p, s^{\prime}-x s\right)\right|_{x=0}$ for $\chi \in \mathcal{S}(\mathbf{R})$. Because $\operatorname{Ad} U_{1}$ preserve Lie bracket, $\mathcal{L}_{1}^{\prime} \equiv\left(\operatorname{Ad} U_{1}\right)\left(\mathcal{L}_{1}\right) \cong \mathcal{L}_{1}$ as a Lie algebra. We obtain a new Lie algebra $\mathcal{L}_{1}^{\prime}$ on $V_{0}$ with generators

$$
X_{f} \otimes I \quad \beta_{s} \otimes I+I \otimes l_{s} \quad f \in C^{\infty}(M), s \in \mathbf{R}
$$

Next step, define $U_{2}: V_{3} \rightarrow V_{3}$ by $\left(U_{2} \Phi\right)(p, s, t) \equiv\left(W_{t} \otimes I \Phi\right)(p, s, t)$. Then $U_{2}$ becomes a linear automorphism on $V_{3}$ and $U_{2} \hat{\pi}(R) U_{2}^{-1}=R \otimes I$, $U_{2} \hat{\Lambda}_{t} U_{2}^{-1}=\hat{\Lambda}_{t}+I \otimes w_{t} \otimes I=I \otimes I \otimes l_{t}+I \otimes w_{t} \otimes I$ for $R \in \mathcal{L}_{1}, t \in \mathbf{R}$. So a Lie algebra $\mathcal{L}_{3} \equiv\left(\operatorname{Ad} U_{2}\right)\left(\operatorname{Ad}\left(U_{1} \otimes I\right)\right)\left(\mathcal{L}_{2}\right)$ with generators

$$
X_{f} \otimes I \otimes I \quad \beta_{s} \otimes I \otimes I+I \otimes l_{s} \otimes I \quad I \otimes I \otimes l_{t}-I \otimes w_{t} \otimes I
$$

for $f \in C^{\infty}(M), t, s, \in \mathbf{R}$ is isomorphic to $\mathcal{L}_{2}$ as a Lie algebra on $V_{3}$. 
Third step, by the fact that the Fourier transformation $T: \mathcal{S}(\mathbf{R}) \rightarrow \mathcal{S}(\mathbf{R})$

$$
(T f)(t) \equiv \frac{1}{\sqrt{2 \pi}} \int_{\mathbf{R}} e^{\sqrt{-1} t s} f(s) d s \quad f \in \mathcal{S}(\mathbf{R})
$$

is bijection and $T l_{t} T^{-1}=w_{t}$, a Lie algebra $\mathcal{L}_{4} \equiv(I \otimes I \otimes T) \mathcal{L}_{3}\left(I \otimes I \otimes T^{-1}\right)$ with generators

$$
X_{f} \otimes I \otimes I \quad \beta_{s} \otimes I \otimes I+I \otimes l_{s} \otimes I \quad I \otimes I \otimes w_{t}+I \otimes w_{t} \otimes I
$$

for $f \in C^{\infty}(M), t, s, \in \mathbf{R}$ is isomorphic to $\mathcal{L}_{2}$ as Lie algebra on $V_{3}$.

Forth step, $U_{3}: V_{3} \rightarrow V_{3}$ is defined by $\left(U_{3} \Phi\right)(p, s, t) \equiv \Phi(p, s-t, t)$ for $\Phi \in V_{3}, p \in M, t, s \in \mathbf{R}$. Then $U_{3}$ becomes a linear automorphism on $V_{3}$ and $U_{3}\left(I \otimes w_{t} \otimes I+I \otimes I \otimes w_{t}\right) U_{3}^{-1}=I \otimes w_{t} \otimes I$ and other generators are invariant under $\operatorname{Ad}\left(U_{3}\right)$. So, $\mathcal{L}_{4} \equiv U_{3} \mathcal{L}_{3} U_{3}^{-1}$ with generators

$$
X_{f} \otimes I \otimes I \quad \beta_{s} \otimes I \otimes I+I \otimes l_{s} \otimes I \quad I \otimes w_{t} \otimes I
$$

$f \in C^{\infty}(M), t, s, \in \mathbf{R}$ is isomorphic to $\mathcal{L}_{2}$. So, naturally it is isomorphic to $\mathcal{L}_{5}$ on $V_{0}$ with generators

$$
X_{f} \otimes I \quad I \otimes l_{s}+\beta_{s} \otimes I \quad I \otimes w_{t} \quad f \in C^{\infty}(M), t, s, \in \mathbf{R}
$$

Then, $\mathcal{L}_{6} \equiv U_{1}^{-1} \mathcal{L}_{5} U_{1}$ on $V_{0}$ has generators

$$
\pi(f) \quad I \otimes l_{s} \quad I \otimes w_{t} \quad f \in C^{\infty}(M), t, s, \in \mathbf{R}
$$

and it is isomorphic to $\mathcal{L}_{2}=\left(\mathfrak{X}_{H} \rtimes_{\beta} \mathbf{R}\right) \rtimes_{\hat{\beta}} \mathbf{R}$.

By the way, $\left[w_{t}, l_{s}\right]=-\sqrt{-1} s t I$ on $\mathcal{S}(\mathbf{R})$ for $s, t \in \mathbf{R}$. ( In quantum mechanics, they are usually denoted by $w_{t}=\sqrt{-1} t \hat{x}$ and $l_{s}=-s \frac{d}{d x}$ on $\mathcal{S}(\mathbf{R})$.) And $\left[I \otimes w_{t}, \pi(f)\right]=0,\left[I \otimes l_{t}, \pi(f)\right]=\left[\Lambda_{t}, \pi(f)\right]=\pi\left(\beta_{t}(f)\right)$. So, Lie algebra generated by $\left\{I \otimes l_{s}, I \otimes w_{t}: t, s, \in \mathbf{R}\right\}$ becomes Heisenberg Lie algebra $H_{1}$ on $V_{0}$.

Here let the Schödinger representation of canonical commutation relation on 1-dimensional harmonic oscillator

$$
a \equiv \frac{1}{\sqrt{2}} I \otimes\left(-\sqrt{-1} w_{1}-l_{1}\right) \quad a^{\dagger} \equiv \frac{1}{\sqrt{2}} I \otimes\left(-\sqrt{-1} w_{1}+l_{1}\right) .
$$

Then $\left[a, a^{\dagger}\right]=I$ and

$$
[a, \pi(f)]=-\frac{1}{\sqrt{2}} \pi\left(\beta_{1}(f)\right) \quad\left[a^{\dagger}, \pi(f)\right]=\frac{1}{\sqrt{2}} \pi\left(\beta_{1}(f)\right)
$$


for $f \in C^{\infty}(M)$. So, let $\delta: H_{1} \rightarrow \operatorname{End}\left(\pi\left(C^{\infty}(M)\right)\right.$ be $\delta_{b}=\operatorname{ad}(b)$ for $b \in H_{1}$, then $\delta$ becomes derivative action. For this derivative action, the semi direct product $\pi\left(C^{\infty}(M)\right) \rtimes_{\delta} H_{1}$ is a Lie algebra with generators

$$
\pi(f) \quad I \otimes w_{t} \quad I \otimes l_{s}
$$

it is just $\mathcal{L}_{6}$. By identification, $\pi\left(C^{\infty}(M)\right) \cong C^{\infty}(M) / \mathrm{C}_{M} \cong \mathfrak{X}_{H}(M)$ $\pi(f) \mapsto-X_{f}$, statements follow.

\section{Discussion}

We obtain a corollary of theorem 6.1 .

Corollary 7.1 For a smooth symplectic flow $\alpha: \mathbf{R} \rightarrow \operatorname{Symp}(M)$ on a symplectic manifold $M$, there is a derivative action $\delta: H_{1} \rightarrow \operatorname{End}\left(\mathfrak{X}_{H}(M)\right)$ such that

$$
\delta_{a} X_{f}=\left.\frac{1}{\sqrt{2}} \frac{d}{d t} X_{\alpha_{t}^{*} f}\right|_{t=0} \quad \delta_{a^{\dagger}} X_{f}=-\left.\frac{1}{\sqrt{2}} \frac{d}{d t} X_{\alpha_{t}^{*} f}\right|_{t=0}
$$

where $H_{1}$ is a Heisenberg algebra with a couple of generators a, $a^{\dagger}$ satisfying Heisenberg commutation relation and $\mathfrak{X}_{H}(M)$ is a Lie algebra of all Hamiltonian vector fields $X_{f}, f \in C^{\infty}(M)$ on $M$.

Difference between original Takesaki duality and infinite case is follows :

$\left(\mathcal{R} \rtimes_{\alpha} \mathbf{R}\right) \rtimes_{\hat{\alpha}} \mathbf{R}$ is separated to $\mathcal{R}$ and $\mathcal{L}\left(L_{2}(\mathbf{R})\right)$ by tensor product. On the other hand, infinitesimal case vector fields and Heisenberg algebra are jointed by derivative action. So, it may be considered that in the case of original Takesaki duality, jointed part is absorbed by strong operator closure of algebraic tensor product $\mathcal{R} \otimes \mathcal{L}\left(L_{2}(\mathbf{R})\right)$.

\section{Appendix}

\section{A $W^{*}$-dynamical system and Takesaki dual- ity}

Let $\mathcal{R}$ be a von Neumann algebra acting on a Hilbert space $\mathcal{H}$. A $\mathrm{W}^{*}$ dynamical system $(\mathcal{R}, G, \alpha)$ is a data such that $G$ is a locally compact group and $\alpha: G \rightarrow \operatorname{Aut}(\mathcal{R})$ is a strong operator continuous action of $G$. 
The crossed product $\mathcal{R} \rtimes_{\alpha} G$ of $(\mathcal{R}, G, \alpha)$ is a von Neumann algebra acting on a Hilbert space $\mathcal{H} \otimes L_{2}(G, \mu) \cong L_{2}(G, \mathcal{H})$ which has generator $\pi(R), \lambda_{g}$ defined by

$$
(\pi(R) \Psi)(h) \equiv\left(\left(\alpha_{h^{-1}}(R) \otimes I\right) \Psi\right)(h) \quad \lambda_{g} \equiv I \otimes L_{g}
$$

for $g, h \in G, R \in \mathcal{R}, \Psi \in \mathcal{H} \otimes L_{2}(G, \mu)$ where $\mu$ is the left Haar measure of $G, L_{g}$ is the left regular representation of $G$.

Assume $G$ is abelian and let $\hat{G}$ be a dual of $G$. Then we can define the second crossed product $\left(\mathcal{R} \rtimes_{\alpha} G\right) \rtimes_{\hat{\alpha}} \hat{G}$ of $(\mathcal{R}, G, \alpha)$. $\left(\mathcal{R} \rtimes_{\alpha} G\right) \rtimes_{\hat{\alpha}} \hat{G}$ is defined by a von Neumann algebra acting on a Hilbert space $\mathcal{H} \otimes L_{2}(G, \mu) \otimes L_{2}(\hat{G}, \nu)$ generated by $\hat{\pi}(S), \hat{\lambda}_{\chi}$ for $S \in \mathcal{R} \rtimes_{\alpha} G, \chi \in \hat{G}$ which defined by

$$
(\hat{\pi}(S) \Phi)(h, \eta) \equiv\left(\left(\hat{\alpha}_{\eta^{-1}}(S) \otimes I\right) \Phi\right)(h, \eta) \quad \hat{\lambda}_{\chi} \equiv I \otimes I \otimes L_{\chi}
$$

for $S \in \mathcal{R} \rtimes_{\alpha} G, \chi, \eta \in \hat{G}, \Phi \in \mathcal{H} \otimes L_{2}(G, \mu) \otimes L_{2}(\hat{G}, \nu)$ where $\nu$ is the left Haar measure of $\hat{G}, L_{\chi}$ is the left regular representation of $\hat{G}$.

Then

Theorem A.1 (Takesaki duality [11])

$$
\left(\mathcal{R} \rtimes_{\alpha} G\right) \rtimes_{\hat{\alpha}} \hat{G} \cong \mathcal{R} \bar{\otimes} \mathcal{L}\left(L_{2}(G, \mu)\right)
$$

where $\mathcal{L}\left(L_{2}(G, \mu)\right)$ is the algebra of all bounded linear operators on $L_{2}(G, \mu)$.

An application of this theorem is the structure theorem of type III-factor.

\section{References}

[1] M.C.Abbati, R.Cirelli, P.Lanzavecchia and A.Manià Pure state of general quantum-mechanical systems as Kähler bundles, Nuovo Cimento B83 (1984) 43-60

[2] R.Cirelli and P.Lanzavecchia Hamiltonian vector fields in quantum mechanics, Nuovo Cimento B79(1983) 271-283

[3] R.Cirelli, P.Lanzavecchia and A.Manià Normal pure states of the Von Neumann algebra of bounded operators as Kähler manifold, J.Phys. A16(1983) 3829-3835 
[4] R.Cirelli, A.Manià and L.Pizzocchero Quantum mechanics as an infinite dimensional Hamiltonian system with uncertainty structure, Part I;Part II, J.Math.Phys 31 (1990) 2891-2897; 2898-2903

[5] R.Cirelli, A.Manià and L.Pizzocchero A functional representation of non-commutative $C^{*}$-algebras, Rev.Math.Phys. Vol. 6, No.5 (1994) 675697.

[6] A. Connes, Non commutative differential geometry, Publ.Math. IHES 62 (1986), 257-360.

[7] A. Connes, Non commutative geometry, Academic Press, Orlando, 1993,

[8] A.T.Fomenko, Symplectic geometry, Gordon and Breach science Publishers (1988)

[9] R.V.Kadison and J.R.Ringrose Fundamentals of the theory of operator algebras $I \sim I V$, Academic Press (1983)

[10] M.A.Rieffel Deformation quantization for Actions of $\mathbf{R}^{d}$, Memories of American Mathematical society vol. 106, No. 506 (1993)

[11] M. Takesaki Duality for crossed products and the structure of von Neumann algebras of type III, Acta Math. 131 (1973), 249-310 